\title{
有机硒催化苯酚选择性氧化制对苯醌的研究
}

\author{
王 芳 $a, b$ 徐 林 ${ }^{a, c}$ 孙 诚 ${ }^{c}$ 徐 清 $a, c$ \\ 黄杰军 $c$ 俞 䂞*,a,c \\ ( ${ }^{a}$ 扬州大学化学化工学院 扬州 22500) \\ ( ${ }^{b}$ 扬州工业职业技术学院 扬州 225127) \\ ( ${ }^{(}$江苏扬农化工集团有限公司 扬州 225009)
}

\begin{abstract}
摘要 苯酚选择性氧化制备对苯醌是一个有重要工业应用价值的反应. 在有机硒催化下, 以过氧化氢为氧化剂氧化苯 酚，高选择性地生成了对苯醌. 与已有技术相比，有机硒催化氧化反应可避免使用金属催化剂，从而避免产物中的金 属残留. 该反应过程清洁, 不产生废弃物, 并可在温和条件下发生. 使用该方法, 对苯醌选择性已经能达到 $91.6 \%$.

关键词 有机硒催化; 苯酚; 氧化; 对苯醌; 对苯二酚
\end{abstract}

\section{Investigation on Preparation of $p$-Benzoquinone through the Organoselenium-Catalyzed Selective Oxidation of Phenol}

\author{
Wang, Fang ${ }^{a, b} \quad \mathrm{Xu}$, Lin $^{a, c} \quad$ Sun, Cheng ${ }^{c} \quad \mathrm{Xu}$, Qing $^{a, c}$ \\ Huang, Jiejun ${ }^{c} \quad$ Yu, Lei $i^{*, a, b, c}$ \\ $\left({ }^{a}\right.$ School of Chemistry and Chemical Engineering, Yangzhou University, Yangzhou 225002) \\ $\left({ }^{b}\right.$ Yangzhou Polytechnology Institute, Yangzhou 225127) \\ ( ${ }^{b}$ Jiangsu Yangnong Chemical Group Co. Ltd., Yangzhou 225009)
}

\begin{abstract}
Selective oxidation of phenol to produce $p$-benzoquinone is an important reaction with good industrial application value. The method for the synthesis of $p$-benzoquinone through the organoselenium-catalyzed selective oxidation of phenol with $\mathrm{H}_{2} \mathrm{O}_{2}$ is reported. Compared with known technologies, organoselenium-catalyzed oxidation reactions avoided the use of metal catalysts and the metal residue in products. The reaction procedures were very clean and were performed under mild conditions. By using this method, the selectivity of $p$-benzoquinone could reach $91.6 \%$ at the maximum.

Keywords organoselenium catalysis; phenol; oxidation; $p$-benzoquinone; hydroquinone
\end{abstract}

苯酚选择性氧化制备对苯醌是一个有重要工业应 用价值的反应. 苯酚是常见的基础化工原料, 目前由异 丙苯氧化裂解法生产, 产量巨大, 价格低廉 ${ }^{[1]}$. 而对苯 醌是合成对苯二酚的中间体. 对苯二酚是重要的高附加 值化工产品，可用于制取医药中间体、黑白显影剂、葱 醌染料、偶氮染料、橡胶防老剂、电极材料、稳定剂和 抗氧剂, 市场前景较好 ${ }^{[2]}$. 目前, 人们对苯酚选择性氧 化制备对苯醌的方法, 做了较多研究. 以过氧化氢为氧 化剂氧化苯酚可避免废弃物生成, 是有较好工业应用前
景的技术方案. 该反应可在铇、钿、钉等金属的复杂配 合物催化下进行 ${ }^{[3]}$, 但催化剂成本较高. 因此, 人们开 始开发各种易回收的非均相无机金属材料来催化这一 过程 ${ }^{[4]}$. 然而, 上述各种金属催化剂, 都会带来产品中 金属残留问题. 因此，改进现有技术，开发无金属参与 的苯酚氧化过程，可以克服这一问题，有着较好的应用 价值.

另一方面, 有机硒催化是一个独特的研究课题. 与 传统金属催化剂相比, 硒催化剂有生态友好 ${ }^{[5]}$ 、价格低

* Corresponding author. E-mail: yulei@yzu.edu.cn.

Received January 12, 2017; revised March 27, 2017; published online April 27, 2017.

Project supported by the National Natural Science Fundation of China (No. 21202141), the Natural Science Foundation of Zhejiang Province for Distinguished Young Scholars (No. LR14B020002), the Priority Academic Program Development of Jiangsu Higher Education Institutions and the High Level Talent Support Project of Yangzhou University.

国家自然科学基金(No. 21202141)、浙江省自然科学基金杰出青年基金(LR14B020002)、江苏省高校优势学科项目及扬州大学高端人才支持计划资助 项目. 
廉以及稳定耐用等优点. 有机硒催化的氧化过程大多使 用清洁的过氧化氢氧化剂, 不产生废弃物, 并且可避免 产品中的金属残留. 目前, 这一领域的研究方兴未艾, 已经逐渐分化为两个方向: 新反应的开发 ${ }^{[6]}$ 与绿色合成 方法学研究 ${ }^{[7,8]}$. 我们团队长期从事有工业应用前景的 绿色化学研究 ${ }^{[7,9]}$, 在有机硒催化领域, 已经取得了一定 的成果, 开发了合成一系列精细化工中间体的绿色合成 方法 ${ }^{[7]}$. 最近, 我们将有机硒催化氧化的方法应用于该 反应中. 本文将报道我们所取得的阶段性研究进展.

\section{1 结果与讨论}

如表 1 所示, 在无催化剂的条件下, 过氧化氢可氧 化苯酚, 但转化率很低, 仅 $21.8 \%$, 并且产物对苯醌的 选择性仅 5.5\%(表 1, Entry 1). 加入二苯基二硒醚做催化 剂后, 苯酚转化率并没有显著提高, 但产物中, 对苯醌 的选择性得到了提升, 为 $23.6 \%$ (表 1, Entry 2). 我们随 后对硒催化剂进行笁选，检验了不同取代基对催化剂性 能的影响. 引入吸电子基团后，二间氯苯基二硒醚所催 化的反应, 对苯醌选择性被显著提高到 $45.9 \%$, 但是苯 酚转化率却降低到 16.8\%(表 1, Entry 3). 而二(3,5-二三 氟甲基苯基)二硒醚的情况则相反，在该化合物催化下， 苯酚转化率可提高到 $44.5 \%$, 而对苯醌选择性则大幅下 降到 7.4\%(表 1, Entry 4). 引入推电子基团后，二对二甲 胺基苯基二硒醚几乎毫无催化活性(表 1, Entry 5), 而有 趣的是，二对甲氧基苯基二硒醚催化该反应是，苯酚转 化率和对苯醌选择性都得到了大幅度的提升, 分别为 $41.5 \%$ 和 45.1\%(表 1, Entry 6). 此外, 我们还检验了脂

表 1 有机硒催化剂的篮选 ${ }^{a}$

Table 1 Screening of the organoselenium catalysts

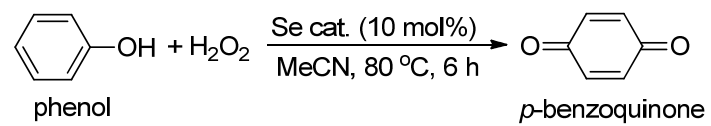

\begin{tabular}{clccc}
\hline Entry & \multicolumn{1}{c}{ Catalyst } & $\mathrm{X}^{b} / \%$ & $\mathrm{~S}^{c} / \%$ & $\mathrm{Y}^{d} / \%$ \\
\hline 1 & \multicolumn{1}{c}{-} & 21.8 & 5.5 & 1.2 \\
2 & $(\mathrm{PhSe})_{2}$ & 22.9 & 23.6 & 5.4 \\
3 & $\left(3-\mathrm{ClC}_{6} \mathrm{H}_{4} \mathrm{Se}\right)_{2}$ & 16.8 & 45.9 & 7.7 \\
4 & {$\left[3,5-\left(\mathrm{CF}_{3}\right)_{2} \mathrm{C}_{6} \mathrm{H}_{3} \mathrm{Se}\right]_{2}$} & 44.5 & 7.4 & 3.3 \\
5 & $\left(4-\mathrm{Me}_{2} \mathrm{NC}_{6} \mathrm{H}_{4} \mathrm{Se}\right)_{2}$ & 6.8 & 0 & 0 \\
6 & $\left(4-\mathrm{MeOC}_{6} \mathrm{H}_{4} \mathrm{Se}\right)_{2}$ & 41.5 & 50.1 & 20.8 \\
7 & $(n-\mathrm{BuSe})_{2}$ & 43.7 & 24.4 & 10.7 \\
8 & $\left(c-\mathrm{C}_{6} \mathrm{H}_{11} \mathrm{Se}\right)_{2}$ & 61.7 & 14.4 & 8.9 \\
\hline
\end{tabular}

${ }^{a}$ A mixture of phenol (3 mmol), $\mathrm{H}_{2} \mathrm{O}_{2}(30 \%$ weight concentration, $15 \mathrm{mmol})$, Se catalyst $(0.3 \mathrm{mmol})$ and $\mathrm{MeCN}(10 \mathrm{~mL})$ was heated at $80{ }^{\circ} \mathrm{C}$ for $6 \mathrm{~h}$; ${ }^{b}$ Conversion rate of phenol; ${ }^{c}$ Selectivity of $p$-benzoquinone detected by HPLC (hydroquinone was generated as one of the by-products); ${ }^{d}$ HPLC-yield of $p$-benzoquinone.

肪族二硒醚在该反应中的催化活性. 研究表明, 使用脂 肪族二正丁基二硒醚和二环己基二硒醚作催化剂, 可显
著提高苯酚转化率，但遗憾的是，对苯醌选择性不佳 (表 1, Entries 7,8).

由以上结果可知，相对于其它二硒醚，二对甲氧基 苯基二硒醚是较好的催化剂. 因此，我们对二对甲氧基 苯基二硒醚催化下苯酚氧化的反应条件进行进一步的 优化. 在化工生产中, 未反应的原料可回收使用, 因此, 反应产物的选择性远比原料的转化率重要. 为了提高该 反应产物对苯醌的选择性, 我们首先尝试了较低温度下 的反应. 研究表明, 反应温度降低到 $60{ }^{\circ} \mathrm{C}$, 虽然苯酚 转化率有所下降, 但产物对苯醌选择性可以显著提高到 $85.9 \%$ (表 2, Entry 2 vs. Entry 1). 进一步降低反应温度并 不能明显提高对苯醌选择性(表 2, Entry 3). 因此, 综合 考虑原料转化率与产物选择性, $60{ }^{\circ} \mathrm{C}$ 是比较适宜的反 应温度. 在此温度下, 我们进一步考察了反应物苯酚的 初始浓度对反应的影响. 实验表明, 苯酚初始浓度为 3 $\mathrm{mol} / \mathrm{L}$ 时较佳(表 2, Entry 2). 降低苯酚初始浓度到 1.5 $\mathrm{mol} / \mathrm{L}$ 并不能明显的优化反应，反而需要消耗更多的溶 剂, 降低产能(表 2, Entry 4). 通过减少溶剂的方法, 提 高苯酚初始浓度后, 苯酚转化率有所提高, 但是对苯醌 选择性明显下降(表 2, Entry 5). 当苯酚初始浓度提高到 $12 \mathrm{~mol} / \mathrm{L}$ 时, 虽然苯酚转化率可高达 92.4\%, 但此时对 苯醌选择性仅有 $24.9 \%$ ，从而使得反应无实用意义(表 2 , Entry 6). 我们随后尝试降低过氧化氢的实用量. 当过氧 化氢的摩尔用量降低到原料的 $250 \%$ 时，反应的原料转 化率与对苯醌选择性, 都得到了提高(表 2, Entry 7). 但 进一步降低过氧化氢用量，会同时降低苯酚转化率与对 苯醌选择性(表 2, Entries 8,9). 实验还表明反应 $6 \mathrm{~h}$ 效果 最佳, 延长反应时间并不能明显地优化反应 (表 2, Entry 10), 但缩短反应时间会导致苯酚转化率下降(表 2 , Entries 11，12). 溶剂篎选实验表明，乙腈是本反应的最 佳溶剂. 反应 $N, N-$ 二甲基甲酰胺(DMF)中进行，苯酚转 化率有所提高, 但对苯醌选择性下降(表 2, Entry 13), 而 在乙醇中, 反应选择性更低(表 2 , Entry 14). 反应在水中 几乎不能很好地进行(表 2, Entry 15). 我们尝试通过提 高温度与增加催化剂的方法来提高反应原料的转化率. 然而, 实验结果表明, 虽然苯酚转化率会被提高, 苯醌 选择性明显下降(表 2, Entries 16, 17). 而在温和条件下, 使用脂肪族二硒醚做催化剂, 亦不能优化反应(表 2 , Entry 18). 因此, 从实际应用角度来说, 使用表 2, Entry 7 的反应条件, 可获得最高的对苯醌选择性, 应当为现 阶段最优条件.

最近, 香港理工大学的 Wong 与 Law 等 ${ }^{[10]}$ 报道了一 则通过自由基机理进行的有机硒催化氧化反应. 因此, 为了证明(或排除)本反应也通过自由基机理进行，我们 尝试在反应中加入 $10 \mathrm{~mol} \%$ 对苯二酚作为自由基清除 
表 2 反应条件的优化 ${ }^{a}$

Table 2 Optimization of the reaction conditions

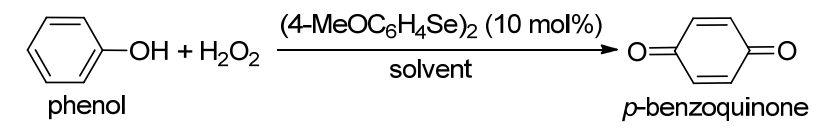

\begin{tabular}{ccccccccc}
\hline Entry & $T /{ }^{\circ} \mathrm{C}$ & $C^{b} /\left(\mathrm{mol} \cdot \mathrm{L}^{-1}\right)$ & $\begin{array}{c}\mathrm{H}_{2} \mathrm{O}_{2} / \\
\text { phenol }^{c}\end{array}$ & \multicolumn{1}{l}{ Solvent $t / \mathrm{h} \mathrm{X}^{d} / \%$} & $\mathrm{~S}^{e} / \%$ & $\mathrm{Y}^{f} / \%{ }^{f}$ \\
\hline 1 & 80 & 3 & 5 & $\mathrm{MeCN}$ & 6 & 41.5 & 50.1 & 20.8 \\
2 & 60 & 3 & 5 & $\mathrm{MeCN}$ & 6 & 15.4 & 85.9 & 13.2 \\
3 & 40 & 3 & 5 & $\mathrm{MeCN}$ & 6 & 9.6 & 88.5 & 8.5 \\
4 & 60 & 1.5 & 5 & $\mathrm{MeCN}$ & 6 & 15.8 & 86.0 & 13.6 \\
5 & 60 & 6 & 5 & $\mathrm{MeCN}$ & 6 & 24.6 & 78.1 & 19.2 \\
6 & 60 & 12 & 5 & $\mathrm{MeCN}$ & 6 & 92.4 & 24.9 & 23.0 \\
7 & 60 & 3 & 2.5 & $\mathrm{MeCN}$ & 6 & 17.1 & 91.6 & 15.7 \\
8 & 60 & 3 & 1.5 & $\mathrm{MeCN}$ & 6 & 17.5 & 78.5 & 13.7 \\
9 & 60 & 3 & 1.2 & $\mathrm{MeCN}$ & 6 & 16.8 & 74.5 & 12.5 \\
10 & 60 & 3 & 2.5 & $\mathrm{MeCN}$ & 8 & 17.2 & 90.6 & 15.6 \\
11 & 60 & 3 & 2.5 & $\mathrm{MeCN}$ & 4 & 13.2 & 89.7 & 11.8 \\
12 & 60 & 3 & 2.5 & $\mathrm{MeCN}$ & 2 & 8.6 & 90.1 & 7.7 \\
13 & 60 & 3 & 2.5 & $\mathrm{DMF}$ & 6 & 27.8 & 60.6 & 16.8 \\
14 & 60 & 3 & 2.5 & $\mathrm{EtOH}$ & 6 & 39.0 & 11.1 & 4.3 \\
15 & 60 & 3 & 2.5 & $\mathrm{H} \mathrm{O}$ & 6 & 6.4 & 0 & 0 \\
16 & 80 & 3 & 2.5 & $\mathrm{MeCN}$ & 6 & 47.4 & 40.1 & 19.0 \\
$17^{g}$ & 60 & 3 & 2.5 & $\mathrm{MeCN}$ & 6 & 56.3 & 49.4 & 27.8 \\
$18^{h}$ & 60 & 3 & 2.5 & $\mathrm{MeCN}$ & 6 & 33.8 & 7.7 & 2.6 \\
\hline
\end{tabular}

${ }^{a} 3$ mmol of phenol was employed; ${ }^{b}$ Initial molar concentration of phenol; ${ }^{c}$ Molar ratio of $\mathrm{H}_{2} \mathrm{O}_{2}$ vs. phenol; ${ }^{d}$ Conversion rate of phenol; ${ }^{e}$ Selectivity of $p$-benzoquinone detected by HPLC; ${ }^{f}$ HPLC-yield of $p$-benzoquinone; ${ }^{g} 20$ mol $\%$ of catalyst was employed; ${ }^{h}(n-\mathrm{BuSe})_{2}$ as catalyst.

剂. 然而实验结果表明, 加入自由基清除剂并不能抑制 反应，反而使得苯酚转化率被显著提高到 $54.3 \%$ (Eq. 1). 由此可见，该反应不可能通过自由基机理发生.

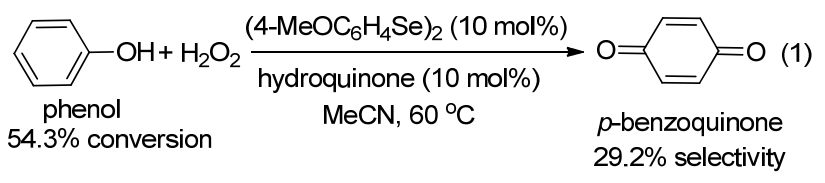

基于文献报道 ${ }^{[7,8,12]}$ 和上述实验结果，我们提出了可 能的反应机理(Scheme 1). 在过氧化氢的氧化下, 二硒
醚可被转化为高价硒活性催化物种 ${ }^{[7,8]}$. 而苯酚首先通 过重排，可生成少量酮式异构体 1 (Scheme 1, path a). 该异构体不稳定，其远离羰基的双键电子云密度较丰 富，可在硒催化下，被过氧化氢氧化，生成二羟基中间 体 $2^{[7 \mathrm{cc}, 7 \mathrm{i}, 7 \mathrm{j}]}$. 类似有机硒催化氧化羰基 $\gamma, \delta$-双键的反应, 我们之前在 $\beta$-紫罗兰酮选择性氧化的工作中，也有所报 道 ${ }^{[7 \mathrm{c}]}$, 从而可以佐证这一步反应机理. 在酸催化下, 中 间体 2 可发生脱水反应，生成含有稳定共轭结构的中间 体 $\mathbf{3}^{[11]}$. 硒催化下，中间体 $\mathbf{3}$ 可被过氧化氢氧化，生成最 终产物对苯醌 ${ }^{[12]}$. 除了上述通过 path a 进行的反应机理 之外, 苯酚还可异构为中间体 4 (Scheme 1, path b). 通 过有机硒催化的烯丙基氧化 ${ }^{[13]}$, 可生成 3 , 最终氧化得 到产物对苯醌. 我们通过高斯软件 G9 程序包, 对反应 中各个中间体的能量进行了分析(各中间体位能标注于 Scheme 1). 计算表明，由于中间体 2 有很低的位能 (一 -459 Hartree), 反应通过 path a 进行的可能性较高.

\section{2 结论}

在二对甲氧基苯基二硒醚催化下，过氧化氢可氧化 苯酚, 高选择性地生成有用化合物对苯醌. 该反应不产 生废弃物, 清洁环保. 产物对苯醌的选择性最高可达 91.6\%, 已经接近工业生成需求. 后续我们将进一步开 发新型有机硒催化剂, 在不降低对苯醌选择性的前提下, 进一步提高反应的转化率, 以提升该技术的应用价值.

\section{3 实验部分}

\section{1 仪器与试剂}

液相色谱仪: 产物定量分析所使用的仪器为 Agilent 1260 液相色谱仪; 色谱条件为: 色谱柱: Agilent Eclipse XDB-C18, 规格 $4.6 \times 250 \mathrm{~mm} \times 5 \mu \mathrm{m}$; 流动相: 乙腈和 $0.15 \%$ 乙酸水溶液; 波长: $254 、 270 \mathrm{~nm}$; 流速: 1.0 $\mathrm{mL} / \mathrm{min}$; 柱温: $35{ }^{\circ} \mathrm{C}$; 进样量 $5.0 \mu \mathrm{L}$. 熔点由 XRC-1 显 微熔点仪测定. 红外谱图由 ENSOR-27 傅里叶红外仪

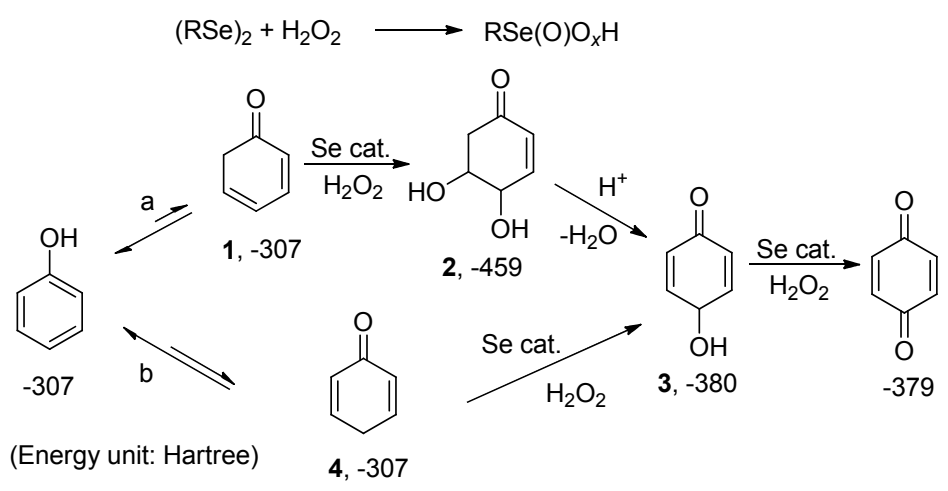

图式 1 可能的反应机理

Scheme 1 Possible mechanisms 
测定. 核磁共振氢谱与碳谱由 Bruker Avance 600 核磁共 振仪测定，以 TMS 为内标.

过氧化氢 $\left(30 \% \mathrm{H}_{2} \mathrm{O}_{2}\right.$, 工业品, 扬农集团)、乙腈 (99\%, A.R., 国药集团)、苯酚 $(98 \%$, A.R., 国药集团)、各 种硒催化剂通过文献方法合成 ${ }^{[7 i]}$.

\section{2 实验方法}

$30 \mathrm{~mL}$ 耐压密封管中加入 $0.282 \mathrm{~g}$ 苯酚 $(3 \mathrm{mmol}$ )、 $1.7 \mathrm{~g}$ 过氧化氢 $(15 \mathrm{mmol}) 、 0.111 \mathrm{~g}$ 二对甲氧基苯基二硒 醚 $(0.3 \mathrm{mmol}) 、 10 \mathrm{~mL}$ 乙腈(溶剂), 氮气置换, 密封反应, 磁力搅拌下控制反应温度 $60{ }^{\circ} \mathrm{C}$, 反应时间 $6 \mathrm{~h}$.

\section{3 表征数据}

对苯醌：固体，m.p. $115 \sim 116{ }^{\circ} \mathrm{C} \quad\left(\mathrm{lit}^{[14]}\right.$. 115 $\left.117{ }^{\circ} \mathrm{C}\right) ;{ }^{1} \mathrm{H}$ NMR $\left(600 \mathrm{MHz}, \mathrm{CDCl}_{3}\right) \delta: 6.80(\mathrm{~s}, 4 \mathrm{H}) ;{ }^{13} \mathrm{C}$ NMR (150 MHz, $\left.\mathrm{CDCl}_{3}\right) \delta$ : 187.2, 136.5; IR (KBr) $v$ : 3080, 1651, 1308, 1100, 952, $890 \mathrm{~cm}^{-1}$; MS (EI, $70 \mathrm{eV}$ ) $m / z(\%): 108\left(81,\left[\mathrm{M}^{+}\right]\right.$), $82(39), 80$ (35), 54 (100).

辅助材料(Supporting Information) 苯酚氧化反应实 验液谱分析细节与液质谱图以及产品红外与核磁共 振谱图. 这些材料可以免费从本刊网站 (http://siocjournal.cn/)上下载.

\section{Referenes}

[1] Bai, W.-B.; Yu, X.-H.; Ding, W.; Xing, R.-T.; Liu, Y. Chem. Ent. Manag. 2013, 3, 84 (in Chinese).

(白卫兵, 余咸旱，丁伟，杏若婷，刘源，化工管理, 2013, 3, 84.)

[2] (a) Kanie, S.; Nishikawa, T.; Ojika, M.; Oba, Y. Sci. Rep. 2016, 6, 24794.

(b) Papa, C. M.; Cesnik, A. J.; Evans, T. C.; Choi, K.-S. Langmuir 2015, 31, 9502 .

(c) Kunitsa, A. A.; Bravaya, K. B. J. Phys. Chem. Lett. 2015, 6, 1053.

(d) Stejskal, J.; Bober, P.; Trchová, M.; Horský, J.; Pilař, J.; Walterová, Z. Synth. Met. 2014, 192, 66.

[3] (a) Gharah, N.; Chakraborty, S.; Mukherjee, A. K.; Bhattacharyya, R. Inorg. Chim. Acta 2009, 362, 1089

(b) Maiti, S. K.; Banerjee, S.; Mukherjee, A. K.; Abdul Malik, K. M.; Bhattacharyya, R. New J. Chem. 2005, 29, 554.

(c) Maiti, S. K.; Abdul Malik, K. M.; Bhattacharyya, R. Inorg. Chem. Commun. 2004, 7, 823.

(d) Wong, W.-K.; Chen, X.-P.; Chik, T.-W.; Wong, W.-Y.; Guo, J.-P.; Lee, F.-W. Eur. J. Inorg. Chem. 2003, 3539.

[4] (a) Cheng, W.-J.; Jiang, Y.-Q.; Xu, X.-Z.; Wang, Y.; Lin, K.-F.; Pescarmona, P. P. J. Catal. 2016, 333, 139.

(b) Inagaki, S.; Tsuboi, Y.; Sasaki, M.; Mamiya, K.; Park, S.; Kubota, Y. Green Chem. 2016, 18, 735.

(c) Wang, B.-R.; Lin, M.; Zhu, B.; Peng, X.-X.; Xu, G.-T.; Shu, X.-T. Catal. Commun. 2016, 75, 69.

(d) Maneesuwan, H.; Tantisriyanurak, S.; Chaisuwan, T.; Wongkasemjit, S. Appl. Catal. A 2015, 504, 448.

[5] (a) Santoro, S.; Azeredo, J. B.; Nascimento, V.; Sancineto, L.; Braga, A. L.; Santi, C. RSC Adv. 2014, 4, 31521.

(b) Freudendahl, D. M.; Santoro, S.; Shahzad, S. A.; Santi, C.; Wirth, T. Angew. Chem., Int. Ed. 2009, 48, 8409.

[6] (a) Guo, R.-Z.; Huang, J.-C.; Huang, H.-Y.; Zhao, X.-D. Org. Lett. 2016, 18, 504. (b) Zhang, X.-L.; Guo, R.-Z.; Zhao, X.-D. Org. Chem. Front. 2015, 2,1334

(c) Cresswell, A. J.; Eey, S. T.-C.; Denmark, S. E. Catal. Nat. Chem. 2015, 7, 146.

(d) Chen, F.; Tan, C. K.; Yeung, Y.-Y. J. Am. Chem. Soc. 2013, 135, 1232.

[7] (a) Jing, X.-B.; Yuan, D.-D.; Yu, L. Adv. Synth. Catal. 2017, 357, 1194.

(b) Wang, Y.-G.; Yu, L.-H.; Zhu, B.-C.; Yu, L. J. Mater. Chem. A 2016, 4, 10828.

(c) Yu, L.; Chen, F.-L.; Ding, Y.-H. ChemCatChem 2016, 8, 1033.

(d) Yu, L.; Bai, Z.-B.; Zhang, X.; Zhang, X.-H.; Ding, Y.-H.; Xu, Q. Catal. Sci. Technol. 2016, 6, 1804.

(e) Zhang, X.; Sun, J.-J.; Ding, Y.-H.; Yu, L. Org. Lett. 2015, 17, 5840 .

(f) Zhang, X.; Ye, J.-Q.; Yu, L.; Shi, X.-K.; Zhang, M.; Xu, Q.; Lautens, M. Adv. Synth. Catal. 2015, 357, 955.

(g) Yu, L.; Ye, J.-Q.; Zhang, X.; Ding, Y.-H.; Xu, Q. Catal. Sci. Technol. 2015, 5, 4830

(h) Yu, L.; Li, H.-Y.; Zhang, X.; Ye, J.-Q.; Liu, J.-P.; Xu, Q.; Lautens, M. Org. Lett. 2014, 16, 1346.

(i) Yu, L.; Wu, Y.-L.; Cao, H.-E.; Zhang, X.; Shi, X.-K.; Luan, J.; Chen, T.; Pan, Y.; Xu, Q. Green Chem. 2014, 16, 287.

(j) Yu, L.; Wang, J.; Chen, T.; Wang, Y.-G.; Xu, Q. Appl. Organomet. Chem. 2014, 28, 652

(k) Yu, L.; Wang, J.; Chen, T.; Ding, K-H.; Pan, Y. Chin. J. Org. Chem. 2013, 33, 1096 (in Chinese)

(俞磊, 王俊, 陈天, 丁克鸿, 潘毅, 有机化学, 2013, 33, 1096.)

[8] (a) Santoro, S.; Santi, C.; Sabatini, M.; Testaferri, L.; Tiecco, M. Adv. Synth. Catal. 2008, 350, 2881.

(b) Sancineto, L.; Tidei, C.; Bagnoli, L.; Marini, F.; Lenardao, E. J.; Santi, C. Molecules 2015, 20, 10496.

[9] (a) Deng, S.-C.; Meng, T.-T.; Xu, B.-L.; Gao, F.; Ding, Y.-H.; Yu, L.; Fan, Y.-N. ACS Catal. 2016, 6, 5807.

(b) Yu, L.; Han, Z.; Ding, Y.-H. Org. Process Res. Dev. 2016, 20, 2124.

(c) Deng, S.-C.; Zhuang, K.; Xu, B.-L.; Ding, Y.-H.; Yu, L.; Fan, Y.-N. Catal. Sci. Technol. 2016, 6, 1772.

(d) Yu, L.; Han, M.-T.; Luan, J.; Xu, L.; Ding, Y.-H.; Xu, Q. Sci. Rep. 2016, 6, 30432.

(e) Xu, L.; Wang, F.; Huang, J.-J.; Xu, Q.; Yu, L.; Fan, Y.-N. Chin. J. Org. Chem. 2016, 36, 2232 (in Chinese).

(徐林, 王芳, 黄杰军, 徐清, 俞否, 范以宁, 有机化学, 2016, 36, 2232.)

(f) Yu, L.; Huang, Y.-P.; Wei, Z.; Ding, Y.-H.; Su, C.-L.; Xu, Q. J. Org. Chem. 2015, 80, 8677.

(g) Yu, L.; Wu, Y.-L.; Chen, T.; Pan, Y.; Xu, Q. Org. Lett. 2013, 15, 144.

(h) Yu, L.; Wang, J.; Cao, H.-E.; Ding, K.-H.; Xu, Q. Chin. J. Org. Chem. 2014, 34, 1986 (in Chinese)

(俞否, 王俊，曹洪恩，丁克鸿，徐清，有机化学, 2014, 34, 1986.)

[10] Jin, W.-W.; Zheng, P.; Wong, W.-T.; Law, G.-L. Adv. Synth. Catal. $\mathbf{2 0 1 7}, 359,1588$

[11] In our previous investigations on the organoselenium-catalyzed oxidation of cyclohexene, small amount of cyclohex-2-en-1-ol and cyclohex-2-en-1-one were also detected as the by-products, indicating the existence of the dehydration step.

[12] van der Toorn, J. C.; Kemperman, G.; Sheldon, R. A.; Arends, I. W. C. E. J. Org. Chem. 2009, 74, 3085.

[13] (a) Niyomura, O.; Cox, M.; Wirth, T. Synlett 2006, 251.

(b) Browne, D. M.; Niyomura, O.; Wirth, T. Org. Lett. 2007, 9, 3169.

(c) Singh, F. V.; Wirth, T. Org. Lett. 2011, 13, 6504.

[14] Patel, S.; Kuanar, M.; Nayak, B. B.; Banichul, H.; Mishra, B. K. Synth. Commun. 2005, 35, 1033.

(Zhao, C.) 